\title{
Effect of magnetic field on electron transport in HgTe/CdTe quantum wells: Numerical analysis
}

\author{
Jiang-chai Chen, ${ }^{1}$ Jian Wang, ${ }^{2}$ and Qing-feng Sun ${ }^{1, *}$ \\ ${ }^{1}$ Beijing National Lab for Condensed Matter Physics and Institute of Physics, Chinese Academy of Sciences, Beijing 100190, China \\ ${ }^{2}$ Department of Physics and The Center of Theoretical and Computational Physics, The University of Hong Kong, Hong Kong, China
}

(Received 5 December 2011; published 2 March 2012)

\begin{abstract}
The effect of magnetic field on electron transport in the inverted band structure of $\mathrm{HgTe} / \mathrm{CdTe}$ quantum well is investigated. Although magnetic field breaks the time-reversal symmetry, the quantum spin Hall effect can still survive at large magnetic field up to $10 \mathrm{~T}$. Moreover, two quantum anomalous Hall-like phases emerge, in which the system only has a spin-up or spin-down edge state at a given sample edge and the edge current is spin polarized. By tuning the Fermi energy, the system can transit between the quantum spin Hall phase and two quantum anomalous Hall-like phases, so the polarized direction of the edge current is well controllable. Thus the spin selectivity can be realized for potential applications of spintronics. Due to the quantum spin and anomalous Hall-like effects, the longitudinal and Hall resistances exhibit quantum plateaus. In addition, at certain magnetic field, some exotic plateaus like $\frac{2}{3}$ fractional quantum Hall effect are also observed, where edge states with the same spin counterpropagate at the one edge. At last, these plateaus are hardly affected by Rashba spin-orbit interaction, Zeeman effect, and Anderson disorder.
\end{abstract}

DOI: 10.1103/PhysRevB.85.125401

PACS number(s): 73.23.-b, 85.75.-d, 73.43.-f

\section{INTRODUCTION}

Recently, much research attention has been attracted to the field of the topological insulator, a topological nontrivial state of matter. ${ }^{1-5}$ The two-dimensional topological insulator, which exhibits the quantum spin Hall (QSH) effect, was theoretically predicted first in graphene and the inverted-band semiconductor $\mathrm{HgTe} / \mathrm{CdTe}$ quantum well. ${ }^{1,3}$ Subsequently, nonzero longitudinal resistance plateau $\frac{1}{2} h / e^{2}$ has been confirmed experimentally. ${ }^{6}$ The two-dimensional topological insulator has an insulating gap in the bulk band structure, but has topologically protected gapless edge states inside the bulk gap. At a given edge of the sample, two edge states with opposite spin polarization counterpropagate. ${ }^{2,7}$ For two edges of the sample, there is a total of four edge states, i.e., there are two pairs of inverted subbands. These edge states are immune to nonmagnetic impurities or geometric perturbations due to the protection of time-reversal symmetry. Soon after, a three-dimensional topological insulator was also proposed theoretically ${ }^{8}$ and observed experimentally in many materials, ${ }^{9}$ such as $\mathrm{Bi}_{1-x} \mathrm{Sb}_{x}, \mathrm{Bi}_{2} \mathrm{Te}_{3}$, and $\mathrm{Bi}_{2} \mathrm{Se}_{3}$.

Based on the QSH state, it was proposed that only one pair of subbands is inverted and the other pair of subbands is normal by some means. ${ }^{10,11}$ In this case, at a given edge, only one edge state, which is spin polarized, survives in the bulk gap. Nonzero quantized Hall resistance and spin-polarized charge current occur without external magnetic field, giving rise to a quantum anomalous Hall (QAH) effect. This spin-polarized current is dissipationless and can have many potential applications in spintronics. But to obtain the QAH effect, time-reversal symmetry must be broken. In previous studies, there are two ways of breaking time-reversal symmetry in order to investigate the QSH/QAH effect. ${ }^{10,12}$ On the one hand, the QAH effect was proposed theoretically in $\mathrm{HgTe} / \mathrm{CdTe}$ quantum well by doping magnetically, and it was shown that the quantized Hall conductance was due to the magnetic moments rather than Landau levels (LLs). ${ }^{10}$ On the other hand, very recently, the QAH and QSH states were found in the Kane and Mele model of two-dimensional honeycomb lattice with broken time-reversal symmetry by the exchange field, where a topological phase transition from a QSH state to a QAH state occurs when the bulk band gap just closes. ${ }^{12}$

In this work, we study the effect of magnetic field on the QSH effect in inverted band structure of the $\mathrm{HgTe} / \mathrm{CdTe}$ quantum well, in which the time-reversal symmetry is broken due to the presence of the magnetic field. We show that the QSH state can survive even in the large magnetic field, similar to the effect of exchange field in the Kane and Mele model. The QSH state can be kept for large magnetic field up to $10 \mathrm{~T}$. Moreover, even at small magnetic field, a gap in spin-down subbands is opened near the bottom of the bulk conduction bands leading to a QAH-like (QAHL) state. In this QAHL state, it has only one spin-up clockwise edge state and the current along the sample edge is spin-up polarized with the longitudinal and Hall resistance plateaus $\left(R_{L}, R_{H}\right)=\left(0, h / e^{2}\right)$. When the magnetic field exceeds a critical value, a gap in spin-up subbands is also opened near the top of bulk valence bands, giving rise to another QAHL state, in which only one spin-down anticlockwise edge state is present and the edge current is spin-down polarized with the longitudinal and Hall resistance plateaus $\left(R_{L}, R_{H}\right)=\left(0,-h / e^{2}\right)$. With the increasing of the magnetic field, the gaps are widened in both spin-up and spin-down subbands, so the ranges of two QAHL states become wide but the range of QSH state becomes narrow. By tuning Fermi energy, the system can transit between the QSH and two QAHL states, thus the direction of spin polarization of edge current can be well controlled. Finally, at very large magnetic field ( $\gtrsim 10 \mathrm{~T}$ ), the QSH state is destroyed and an insulator state appears, but two QAHL states still exist for a very wide range of Fermi energy.

Furthermore, at the upmost part of the bulk gap, four pairs of longitudinal and Hall resistance plateaus (including a pair of QAHL plateaus) are clearly exhibited at the small magnetic field (about $0.2 \mathrm{~T}$ ) due to the QSH edge state and LL edge states. In particular, a pair of longitudinal and Hall resistance plateaus $R_{L}=\frac{2}{9} h / e^{2}$ and $R_{H}=\frac{1}{3} h / e^{2}$ are found in which the spin down has both clockwise and anticlockwise 
edge states. This means that the currents with the same spin and opposite momentum coexist at a given edge, like $\frac{2}{3}$ fractional quantum Hall effect in the two-dimensional electron gas having strong electron-electron interaction. Moreover, the effects of Rashba spin-orbit interaction and Anderson disorder are also investigated. All of the above results for the clean sample, including resistance plateaus and the QSH and QAHL states, are hardly affected by Rashba spin-orbit interaction, Zeeman effect, and Anderson disorder. Since the Hall and longitudinal resistances in $\mathrm{HgTe} / \mathrm{CdTe}$ quantum well under magnetic field has been successfully measured ${ }^{6,13-15}$ the predicted phenomena should easily be observed in the experiment.

The rest of the paper is organized as follows. In Sec. II, we describe the model and give the details of our calculations. In Sec. III, the numerical results are given. Finally, a brief conclusion is presented in Sec. IV.

\section{MODEL AND CALCULATION}

The $\mathrm{HgTe} / \mathrm{CdTe}$ quantum well under a perpendicular magnetic field is modeled as a six-terminal device shown in the inset of Fig. 1(b). We neglect the terms $\propto k^{2}$, which is small near the $\Gamma$ point. ${ }^{16}$ Bulk inversion asymmetry is also omitted because the subband mixing is suppressed by strong magnetic field. ${ }^{17}$ Experimentally, Zeeman splitting is very small in $\mathrm{HgTe} / \mathrm{CdTe}$ quantum well if the magnetic field is not very strong, e.g., smaller than $10 \mathrm{~T} .{ }^{13}$ Here we first neglect the Zeeman effect and it will be discussed at last. So we start from the effective Hamiltonian in $\mathrm{HgTe} / \mathrm{CdTe}$ quantum well with magnetic field and Rashba spin-orbit interaction. Its discrete form in the tight-binding representation of a square lattice is given by ${ }^{18-20}$

$$
H=\sum_{\mathbf{i}}\left[\varphi_{\mathbf{i}}^{\dagger} V \varphi_{\mathbf{i}}+\left(\varphi_{\mathbf{i}}^{\dagger} T_{\hat{\mathbf{x}}} \varphi_{\mathbf{i}+\hat{\mathbf{x}}}+\varphi_{\mathbf{i}}^{\dagger} T_{\hat{\mathbf{y}}} \varphi_{\mathbf{i}+\hat{\mathbf{y}}}+\text { H.c. }\right)\right],
$$

where $\mathbf{i}=\left(\mathbf{i}_{x}, \mathbf{i}_{y}\right)$ is the site index, $\hat{\mathbf{x}}$ and $\hat{\mathbf{y}}$ are unit vectors along $x$ and $y$ directions. $\varphi_{\mathbf{i}}=\left(a_{\mathbf{i}}, b_{\mathbf{i}}, c_{\mathbf{i}}, d_{\mathbf{i}}\right)^{T}$ represents the four annihilation operators of electron on the site $\mathbf{i}$ at the special states $|s, \uparrow\rangle,\left|p_{x}+i p_{y}, \uparrow\right\rangle,|s, \downarrow\rangle$, and $\left|-\left(p_{x}-i p_{y}\right), \downarrow\right\rangle$, respectively. $V=\operatorname{diag}\left(E_{s}, E_{p}, E_{s}, E_{p}\right)$ is the matrix of on-site energy with $E_{s / p}=C \pm M-4(D \pm B) / a^{2}$;

$$
T_{\hat{\mathbf{x}}}=\left(\begin{array}{cccc}
V_{s s} & V_{s p} & V_{R} & 0 \\
-V_{s p}^{*} & V_{p p} & 0 & 0 \\
-V_{R}^{*} & 0 & V_{s s} & V_{s p}^{*} \\
0 & 0 & -V_{s p} & V_{p p}
\end{array}\right) \exp \left(i \phi_{\mathbf{i}, \mathbf{i}+\hat{\mathbf{x}}}\right)
$$

and

$$
T_{\hat{\mathbf{y}}}=\left(\begin{array}{cccc}
V_{s s} & i V_{s p} & -i V_{R} & 0 \\
i V_{s p}^{*} & V_{p p} & 0 & 0 \\
-i V_{R}^{*} & 0 & V_{s s} & -i V_{s p}^{*} \\
0 & 0 & -i V_{s p} & V_{p p}
\end{array}\right) \exp \left(i \phi_{\mathrm{i}, \mathrm{i}+\hat{\mathbf{y}}}\right)
$$

are the hopping matrices along $x$ and $y$ directions, respectively, with $V_{s s / p p}=(D \pm B) / a^{2}, V_{s p}=-i A /(2 a)$ and $V_{R}=$ $\alpha /(2 a)$. Here, $a$ is the lattice constant, $\alpha$ is the strength of Rashba spin-orbit interaction, and $A, B, C, D$, and $M$ are the system parameters, which can be experimentally controlled. The effect of the perpendicular magnetic field $B_{\perp}$ is included

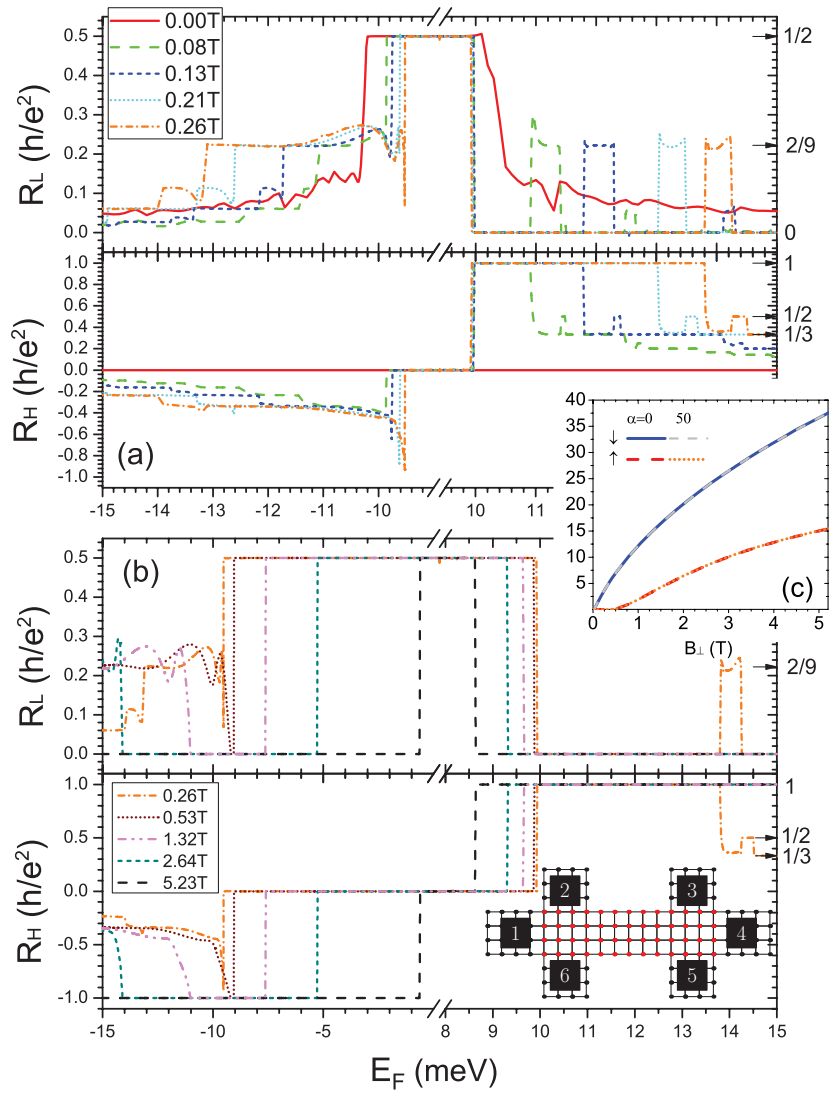

FIG. 1. (Color online) Longitudinal and Hall resistances as a function of Fermi energy $E_{F}$ with fixed magnetic field $B_{\perp}$ : (a) small and (b) large magnetic fields. Inset of (b): the schematic of six-terminal device. (c) The widths of gaps in the spin-up ( $\uparrow)$ and spin-down $(\downarrow)$ subbands when varying the magnetic field $B_{\perp}$ with and without Rashba spin-orbit interaction.

by the phase $\phi_{\mathbf{i}, \mathbf{j}}=\int_{\mathbf{i}}^{\mathbf{j}} \mathbf{A} \cdot \mathbf{d} \mathbf{l} / \phi_{0}$ in the hopping matrices where $\mathbf{A}=\left(-y B_{\perp}, 0,0\right)$ is the vector potential and $\phi_{0}=\hbar / e$.

From the Landauer-Büttiker formula at zero temperature, ${ }^{21}$ the current flowing into terminal $p$ is given by

$$
I_{p}=\frac{e^{2}}{h} \sum_{q(\neq p)} T_{p q}\left(E_{F}\right)\left(V_{p}-V_{q}\right) .
$$

Here, $T_{p q}\left(E_{F}\right)=\operatorname{Tr}\left[\boldsymbol{\Gamma}_{p} \mathbf{G}^{r} \Gamma_{q} \mathbf{G}^{a}\right](p, q=1,2, \ldots, 6$ and $p \neq$ $q$ ) is the transmission coefficient from terminal $q$ to terminal $p$ at Fermi energy $E_{F}, \boldsymbol{\Gamma}_{p}\left(E_{F}\right)=i\left[\boldsymbol{\Sigma}_{p}^{r}\left(E_{F}\right)-\boldsymbol{\Sigma}_{p}^{a}\left(E_{F}\right)\right]$ are the linewidth functions, $\mathbf{G}^{r}\left(E_{F}\right)=\left[\mathbf{G}^{a}\right]^{\dagger}=1 /\left[E_{F}-\mathbf{H}_{c}-\right.$ $\left.\sum_{p=1}^{6} \boldsymbol{\Sigma}_{p}^{r}\right]$ are the retarded and advanced Green's functions, and $\mathbf{H}_{c}$ is the Hamiltonian of the region, which includes two cross parts and the region between them [see the inset of Fig. 1(b) with red grid]. The retarded self-energy $\boldsymbol{\Sigma}_{p}^{r}\left(E_{F}\right)$ due to the coupling to the terminal $p$ can be calculated numerically. ${ }^{22}$ We applied a bias $V$ across terminal 1 and terminal 4 , and the currents in the voltage probes (terminals 2 , 3,5 , and 6) are set to zero. Then from the Landauer-Büttiker formula, the voltages $V_{2}, V_{3}, V_{5}$, and $V_{6}$ of the voltage probes and the currents $I_{1}$ and $I_{4}$ can be calculated. Owing to current conservation, $I_{1}=-I_{4} \equiv I_{14}$. Finally, the longitudinal and Hall resistances are given by $R_{L} \equiv\left(V_{2}-V_{3}\right) / I_{14}$ and $R_{H} \equiv\left(V_{2}-V_{6}\right) / I_{14}$, respectively. 
In the following numerical calculation, the parameters of the $\mathrm{HgTe} / \mathrm{CdTe}$ quantum well is chosen as $A=364.5 \mathrm{meV} \mathrm{nm}$, $B=-686 \mathrm{meV} \mathrm{nm}^{2}, C=0, D=-512 \mathrm{meV} \mathrm{nm}^{2}$, and $M=$ $-10 \mathrm{meV}$, which correspond to the realistic well with $7 \mathrm{~nm}$ of thickness ${ }^{15}$ exceeding the critical thickness $6.3 \mathrm{~nm}$ to invert the band. So the quantum well without magnetic field is in the topological phase. The lattice constant is adopted as $a=5$ $\mathrm{nm}$. The width of ribbon with square lattice is $a(2 N-1)=$ $495 \mathrm{~nm}$ with $N=50$, and the widths of voltage terminals 2 , $3,5,6$ are the same as those of ribbon. The distance between terminal 2 and terminal 3 is $a L=100 \mathrm{~nm}$ with $L=20$.

\section{NUMERICAL RESULTS AND ANALYSIS}

First, we consider the ideal six-terminal system under the perpendicular magnetic field such that the edge state survives. Hereafter the symbol $\left(n_{c \uparrow}, n_{a \uparrow} ; n_{c \downarrow}, n_{a \downarrow}\right)$ denotes the number of edge states with different combinations. Here, $\uparrow(\downarrow)$ refers the spin up (down) and the subscript $c(a)$ denotes the clockwise (anticlockwise) chirality of edge state. So $n_{c}=n_{c \uparrow}+n_{c \downarrow}$ and $n_{a}=n_{a \uparrow}+n_{a \downarrow}$ are the total numbers for clockwise and anticlockwise edge states, respectively. Due to the chiral nature of the edge state, we have $T_{p+1, p}=T_{16}=n_{c}, T_{p, p+1}=T_{61}=$ $n_{a},(p=1,2, \ldots, 5)$ and the rest of transmission coefficient $T_{p q}=0$. By substituting these transmission coefficients into Eq. (4), the plateaus of the longitudinal and Hall resistances can be analytically obtained as

$$
R_{L}=\frac{n_{c} n_{a}}{n_{c}^{3}+n_{a}^{3}} \frac{h}{e^{2}}, \quad R_{H}=\frac{n_{c}-n_{a}}{n_{c}^{2}+n_{a}^{2}-n_{c} n_{a}} \frac{h}{e^{2}} .
$$

In Fig. 1, the longitudinal and Hall resistance are investigated as we vary Fermi energy with fixed magnetic field and no Rashba spin-orbit interaction. When there is no magnetic field [see in Fig. 1(a)], the longitudinal and Hall resistances are $\frac{1}{2} h / e^{2}$ and zero, respectively, which indicate that QSH effect occurs because of two edge states in the bulk gap $(|E|<|M|)$ of inverted bands. Except for this resistance plateau there is no other plateau. For small magnetic field, besides the plateaus of QSH effect, four pairs of longitudinal and Hall resistance plateaus appear clearly for $E_{F}>|M|$ in Fig. 1(a). The first pair of resistance plateaus are $\left(R_{L}, R_{H}\right)=\left(0, h / e^{2}\right)$. This pair of plateaus become wider with the increasing of magnetic field. For instance, the width of the plateaus is $0.9 \mathrm{meV}$ for $B_{\perp}=0.08 \mathrm{~T}$, but $3.8 \mathrm{meV}$ for $B_{\perp}=0.26 \mathrm{~T}$. If the Fermi energy moves toward larger value, the second pair of resistance plateaus occur with $\left(R_{L}, R_{H}\right)=\left(\frac{2}{9} h / e^{2}, \frac{1}{3} h / e^{2}\right)$. The range of quantized plateau for this pair is the largest for particular magnetic field around $0.13 \mathrm{~T}$. The wider the quantum well is, the finer the plateaus are. Following these plateaus, the third pair of resistance plateaus are $R_{L}=0$ and $R_{H}=\frac{1}{2} h / e^{2}$, and the fourth pair are $R_{L}=0$ and $R_{H}=\frac{1}{3} h / e^{2}$. On the side of $E_{F}<-|M|$, we also see some plateaus due to Landau levels (LLs), but these plateaus are quite vague.

In Fig. 1(b) for large magnetic field, the regime of QSH effect with $\left(R_{L}, R_{H}\right)=\left(\frac{1}{2} e^{2} / h, 0\right)$ still exists, but its range is reduced as the magnetic field increases. For example, the width of regime decreases from $20 \mathrm{meV}$ for $B_{\perp}=0.26 \mathrm{~T}$ to $9 \mathrm{meV}$ for $B_{\perp}=5.23 \mathrm{~T}$. On the side of $E_{F}>0$, compared with small magnetic field, the first pair of plateaus with $\left(R_{L}, R_{H}\right)=\left(0, h / e^{2}\right)$ widen clearly, and other resistance pairs
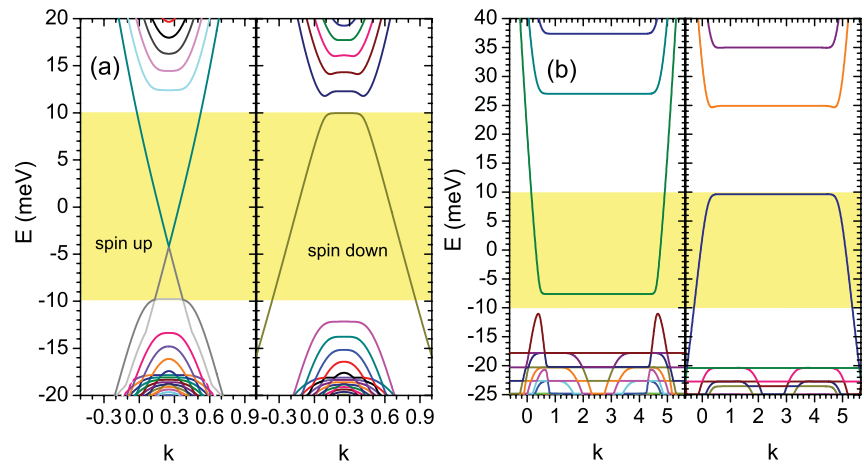

FIG. 2. (Color online) The energy band structures of $\mathrm{HgTe} / \mathrm{CdTe}$ quantum well with (a) $B_{\perp}=0.13 \mathrm{~T}$ (small magnetic field) and (b) $B_{\perp}=1.3 \mathrm{~T}$ (large magnetic field). The left and right halves of each plot denote the spin-up and spin-down subbands, respectively. The gray/yellow region is bulk gap (i.e., $|E|<|M|$ ) without magnetic field.

are pushed into larger energy. What is more, on the side of $E_{F}<0$, a new pair of resistance plateaus, $\left(R_{L}, R_{H}\right)=$ $\left(0,-h / e^{2}\right)$, appear and become wider as magnetic field increases. From Fig. 1(b), we can clearly see that this plateau pair does not exist at $B_{\perp}=0.26 \mathrm{~T}$, while for $B_{\perp}=0.53 \mathrm{~T}$ the plateaus appear. So it indicates a critical magnetic field $B_{c}$. When magnetic field is larger than $B_{c}$, the resistance plateaus with $\left(R_{L}, R_{H}\right)=\left(0,-h / e^{2}\right)$ occur. In Fig. 1(c), we find $B_{c} \approx 0.5 \mathrm{~T}$.

To understand the behaviors of Fig. 1, we plot the energy-band structure of $\mathrm{HgTe} / \mathrm{CdTe}$ quantum well with small and large magnetic fields in Fig. 2. In the Hamiltonian of Eq. (1), no coupling between two spin spaces exists in the absence of Rashba interaction. So the spin subbands can be depicted separately. In Fig. 2(a) for band structure of small magnetic field, the regime of QSH effect is still held for $|E| \lesssim|M|$, where two edge states counterpropagate at one edge. One of edge states, such as spin up, is clockwise and the other, spin down, is anticlockwise. Thus $\left(n_{c \uparrow}, n_{a \uparrow} ; n_{c \downarrow}, n_{a \downarrow}\right)=(1,0 ; 0,1)$ and the longitudinal and Hall resistances $\left(R_{L}, R_{H}\right)=\left(\frac{1}{2} h / e^{2}, 0\right)$, which is consistent with the previous theoretical and experimental results. ${ }^{3,6}$ On the side of $E>|M|$, a gap is opened in spin-down subbands at the bottom of bulk conduction bands, which indicates a transition from QSH state to quantum Hall $(\mathrm{QH})$ state. $^{23}$ If Fermi energy is located in this gap, the remaining edge state is only spin up with clockwise chirality. Thus edge-state numbers $\left(n_{c \uparrow}, n_{a \uparrow} ; n_{c \downarrow}, n_{a \downarrow}\right)=(1,0 ; 0,0)$, leading to the first pair of the resistance plateaus $\left(R_{L}, R_{H}\right)=\left(0, h / e^{2}\right)$, which can be regarded as a result of special $\mathrm{QH}$ effect. This current is spin polarized and dissipationless, like the spin polarization in the QAH effect mentioned in Sec. I. In this regime, the system has the behavior of the QAH system ${ }^{10}$ with the spin Chern numbers $C_{+}=1$ and $C_{-}=0$, so we name it as the QAHL regime. Besides, on the topmost part of the gap in spin-down subbands, there are two dips that result in the edge-state numbers $\left(n_{c \uparrow}, n_{a \uparrow} ; n_{c \downarrow}, n_{a \downarrow}\right)=(1,0 ; 1,1)$ and the plateaus of $\left(R_{L}, R_{H}\right)=\left(\frac{2}{9} h / e^{2}, \frac{1}{3} h / e^{2}\right)$, which will be unveiled with corresponding wave functions in Fig. 5. If Fermi energy increases furthermore and just moves out of the dips of 
spin-down subbands, it enters into a narrow energy region [a similar region is seen more clearly in Fig. 4(b)] where the edge-state numbers are $(1,0 ; 1,0)$ leading to the third pair of plateaus $\left(R_{L}, R_{H}\right)=\left(0, \frac{1}{2} h / e^{2}\right)$. In this regime, both the edge states of spin up and spin down are clockwise. If the Fermi energy continues to increase, another spin-up Hall edge state that is clockwise also participates in the conducting, so edge states are $(2,0 ; 1,0)$ now, which denote the fourth pair of plateaus of $\left(R_{L}, R_{H}\right)=\left(0, \frac{1}{3} h / e^{2}\right)$. On the side of $E<-|M|$, LLs begin to be formed in the small magnetic field (e.g., $\sim 0.2 \mathrm{~T}$ ). Accordingly, some vague plateaus appear in Fig. 1(a) for $E_{F}<-|M|$.

For the energy-band structure of large magnetic field in Fig. 2(b), except for the gap of spin-down subbands, the spin-up subbands also open a gap. The gaps in both spin-up and spin-down subbands become wider with the increase of magnetic field. As a result, the QSH regime with the plateaus $\left(R_{L}, R_{H}\right)=\left(\frac{1}{2} h / e^{2}, 0\right)$ is reduced, e.g., from $20 \mathrm{meV}$ at $B_{\perp}=0.13 \mathrm{~T}$ to $17.5 \mathrm{meV}$ at $B_{\perp}=1.3 \mathrm{~T}$. Meanwhile, on the side of $E>|M|$, due to the widening of the gap of spin-down subbands, the QAHL regime with $\left(R_{L}, R_{H}\right)=\left(0, h / e^{2}\right)$ and the spin-polarized edge current can exist in a very large energy range, $\approx 14.5 \mathrm{meV}$ at $B_{\perp}=1.3 \mathrm{~T}$ [compared with $1.8 \mathrm{meV}$ for small $B_{\perp}=0.13 \mathrm{~T}$ in Fig. 2(a)]. The second pair of plateaus $\left(\frac{2}{9} h / e^{2}, \frac{1}{3} h / e^{2}\right)$ are distorted and nearly disappear due to the mixing between the QSH edge state and the LL edge state. But the third and fourth pairs of plateaus resulting from the bulk LLs are more visible under the large magnetic field. On the side of $E<-|M|$, a gap of spin-up subbands is opened as well. When Fermi energy is located in this subband gap, there only exists an anticlockwise spindown edge state with $\left(n_{c \uparrow}, n_{a \uparrow} ; n_{c \downarrow}, n_{a \downarrow}\right)=(0,0 ; 0,1)$ and the resistance plateaus $\left(R_{L}, R_{H}\right)=\left(0,-h / e^{2}\right)$. In this gap regime, the edge current is spin polarized and dissipationless, and it likes the QAH effect with the spin Chern numbers $C_{+}=0$ and $C_{-}=-1$ because only the anticlockwise spin-down edge state contributes. Hence, under strong magnetic field, the system can be controlled between the QSH $\left(C_{ \pm}= \pm 1\right)$ and two QAHL $\left(C_{+}=1\right.$ and $C_{-}=0 ; C_{+}=0$ and $\left.C_{-}=-1\right)$ phases by tuning the Fermi energy. Notice that the current has the different spinpolarization directions in the two QAHL phases, which means we can control the direction of spin polarization by simply tuning the Fermi energy. In Fig. 1(c), we show the spin-up and spin-down subband gaps versus the magnetic field $B_{\perp}$. With the increase of magnetic field, the gaps (i.e., the ranges of the QAHL effects) widen. The gap of spin-down subbands opens with the presence of the magnetic field, but there is a critical magnetic field $B_{c}$ for the opening of the spin-up gap.

Next, the resistances $\left(R_{L}, R_{H}\right)$ with fixed Fermi energy as a function of the magnetic field are investigated in Fig. 3.
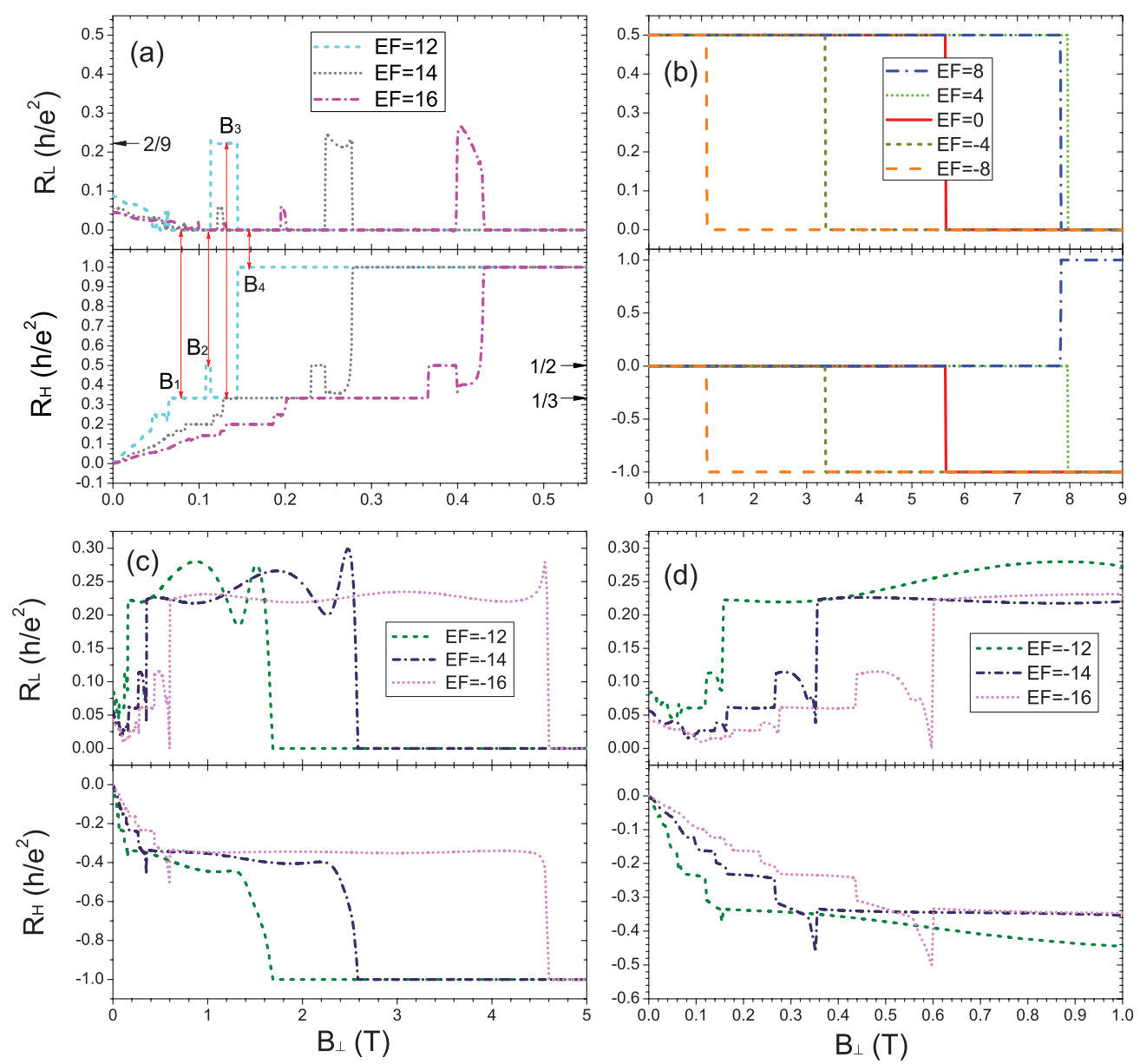

FIG. 3. (Color online) The resistances $R_{L}$ and $R_{H}$ vs magnetic field $B_{\perp}$ with different Fermi energies: (a) $E_{F}>|M|$, (b) $|M|>E_{F}>-|M|$, and (c) $-|M|>E_{F}$. (d) is an amplification of (c) for small magnetic field. 
The Fermi energy $E_{F}$ is divided into three regions: $E_{F}>$ $|M|,|M|>E_{F}>-|M|$, and $-|M|>E_{F}$. For the region of $E_{F}>|M|$ [see Fig. 3(a)], because of the carriers being electron, the Hall resistance is positive and linearly increases for small magnetic field $B_{\perp}$, which is in good agreement with the experiment results. ${ }^{6,13}$ Then with the magnetic field increasing further, LLs are formed gradually and Hall plateaus appear. There are four pairs of longitudinal and Hall resistances $\left(R_{L}, R_{H}\right)=\left(0, \frac{1}{3} h / e^{2}\right),\left(0, \frac{1}{2} h / e^{2}\right),\left(\frac{2}{9} h / e^{2}, \frac{1}{3} h / e^{2}\right)$, and $\left(0, h / e^{2}\right)$, which have been mentioned in Fig. 1(a). These plateaus are clearly shown, especially for $E_{F}=12 \mathrm{meV}$. The $\left(\frac{2}{9} h / e^{2}, \frac{1}{3} h / e^{2}\right)$ plateaus are exhibited perfectly only when $E_{F}$ is slightly higher than $|M|$ (e.g., $E_{F}=12 \mathrm{meV}$ ), and have a departure for the high Fermi energy $E_{F}$. The other three pairs of resistance plateaus can be held in all ranges of Fermi energy $E_{F}>|M|$. The higher the Fermi energy is, the better and wider the plateaus are.

The corresponding energy bands are depicted for four resistance-plateau pairs in Fig. 4. As the magnetic field increases, the conduction bands move toward higher energy for both spin-up and spin-down subbands. At $B_{\perp}=0.08 \mathrm{~T}$ [see Fig. 4(a)], the edge state numbers at $E_{F}=12 \mathrm{meV}$ are $(2,0 ; 1,0)$ and its plateaus are $\left(R_{L}, R_{H}\right)=\left(0, \frac{1}{3} h / e^{2}\right)$ [see Fig. 3(a)]. At $B_{\perp}=0.11 \mathrm{~T}$ [see Fig. 4(b)], edge states are $(1,0 ; 1,0)$ on the crossing of bands with Fermi energy, so the plateaus with $\left(R_{L}, R_{H}\right)=\left(0, \frac{1}{2} h / e^{2}\right)$ are shown in Fig. 3(a). At $B_{\perp}=0.13 \mathrm{~T}$ [see Fig. 4(c)], a mixture between the QSH edge state and electron LL edge states occurs, leading to $\left(n_{c \uparrow}, n_{a \uparrow} ; n_{c \downarrow}, n_{a \downarrow}\right)=(1,0 ; 1,1)$ and $\left(R_{L}, R_{H}\right)=$ $\left(\frac{2}{9} h / e^{2}, \frac{1}{3} h / e^{2}\right)$. At $B_{\perp}=0.16 \mathrm{~T}$ [see Fig. 4(d)], Fermi energy
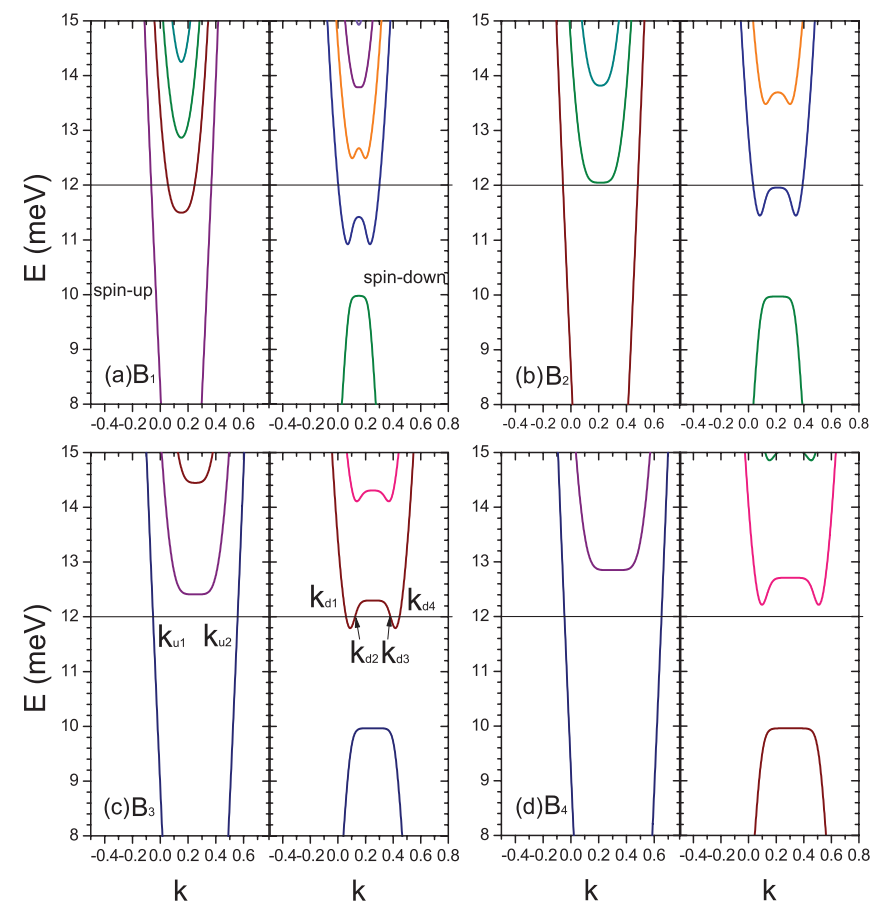

FIG. 4. (Color online) The energy-band structures of $\mathrm{HgTe} / \mathrm{CdTe}$ quantum well around Fermi energy $E_{F}=12 \mathrm{meV}$ with different magnetic field selected from Fig. 3(a): (a)-(d) correspond $B_{\perp}=0.08$, $0.11,0.13$, and $0.16 \mathrm{~T}$, respectively. The left and right halves of each plot denote the spin up and spin down.

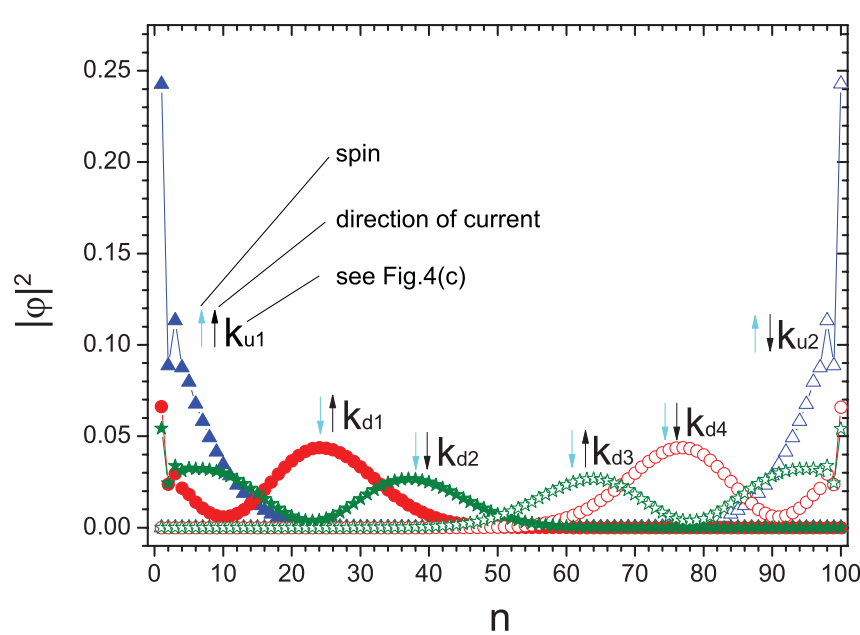

FIG. 5. (Color online) The corresponding wave functions of six momentum points owing to the crossing of Fermi energy with energy bands in Fig. 4(c). The first arrow denotes the spin type, and the second arrow denotes the current direction of edge state. Here, the chirality of spin-up edge states is adopted as clockwise direction.

$E_{F}=12 \mathrm{meV}$ is located in the gap of spin-down subbands, in which the system is in the QAHL regime with the edge states being $(1,0 ; 0,0)$ and $\left(R_{L}, R_{H}\right)=\left(0, h / e^{2}\right)$.

To understand further $\left(R_{L}, R_{H}\right)=\left(\frac{2}{9} h / e^{2}, \frac{1}{3} h / e^{2}\right)$ plateaus, the corresponding wave functions of the momentums $k_{u 1}$, $k_{u 2}, k_{d 1}, k_{d 2}, k_{d 3}$, and $k_{d 4}$ [see Fig. 4(c)] are depicted in Fig. 5. The wave functions of $k_{u 1}, k_{d 1}$, and $k_{d 2}$ are located on the left boundary and the others are on the right side, which clearly show that they indeed are the edge states. While for a wide device, these edge states locating on one boundary are separated far from the opposite boundary. Spin up only has one clockwise edge state (the state $k_{u 1}$ ), while spin down has one clockwise (the state $k_{d 1}$ ) and one anticlockwise (the state $k_{d 2}$ ). Here the anticlockwise state $k_{d 2}$ is the QSH edge state and the clockwise state $k_{d 1}$ is the bulk electron LL edge state. Thus the edge state numbers are $(1,0 ; 1,1)$, giving rise to resistance plateaus $\left(\frac{2}{9} h / e^{2}, \frac{1}{3} h / e^{2}\right)$. Here, it is exotic for spin-down edge states that the edge states with the same spin counterpropagate on the same boundary with little mixing. A similar phenomenon occurs in (e.g., 2/3) fractional quantum Hall effect only, in which there exists strong electron-electron interaction. ${ }^{24,25}$

In the following, we investigate the resistance plateaus as a function of magnetic field with Fermi energy in the range of $|M|>E_{F}>-|M|$. This range is the QSH regime while without the magnetic field. The results are shown in Fig. 3(b). With the increasing of the magnetic field, the regime of the QSH effect, i.e., the width of the resistance plateaus $\left(R_{L}, R_{H}\right)=\left(\frac{1}{2} h / e^{2}, 0\right)$ in Figs. 1(a) and 1(b), is narrowed and finally disappears when the magnetic field is large enough ( $>10 \mathrm{~T})$. For Fermi energy $E_{F}>E_{D}\left(E_{D} \approx 7.5 \mathrm{meV}\right.$ is the Dirac point at $B_{\perp}=0$ ), the system enters from the QSH phase into the QAHL phase of $C_{+}=1$ and $C_{-}=0$ at the large magnetic field, in which there is only a clockwise spin-up edge state and the corresponding resistance plateaus are $\left(R_{L}, R_{H}\right)=\left(0, h / e^{2}\right)$. On the other hand, if Fermi energy $E_{F}<E_{D}$, the system enters into the QAHL phase of $C_{+}=0$ and $C_{-}=-1$ at large $B_{\perp}$, in which only an anticlockwise 


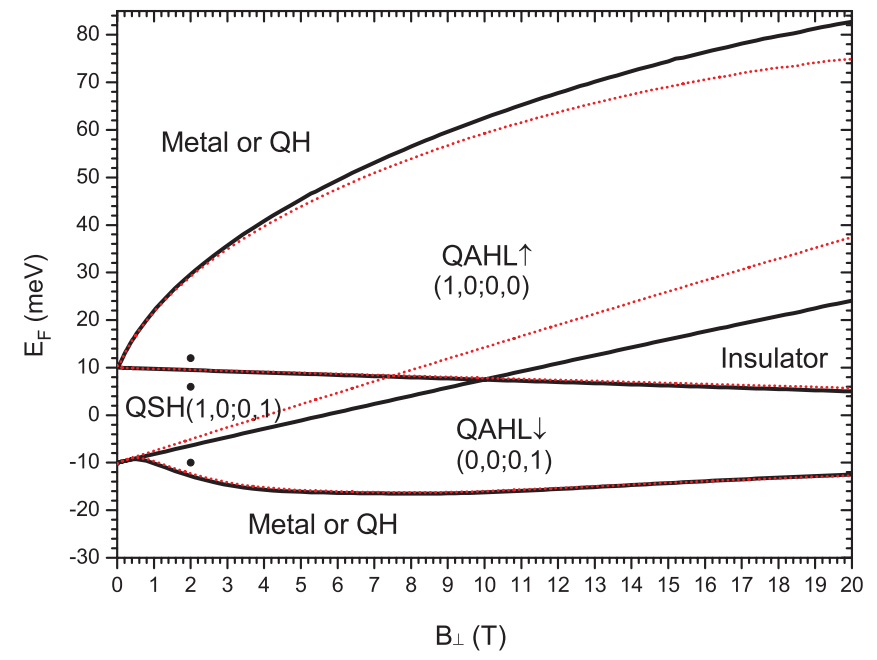

FIG. 6. (Color online) Phase diagram in the $E_{F}-B_{\perp}$ plane without (black solid) and with (red dotted) Zeeman effect.

spin-down edge state exists and the resistance plateaus are $\left(R_{L}, R_{H}\right)=\left(0,-h / e^{2}\right)$. Also, it is shown that the system entering the QAHL phase occurs at larger magnetic field if Fermi energy $E_{F}$ is closer to $E_{D}$. For instance, from Fig. 3(b), we can see that the phase transition occurs at magnetic field $B_{\perp}=1.1,3.4,5.6$, and $8.0 \mathrm{~T}$, respectively, while $E_{F}=-8$, $-4,0$, and $4 \mathrm{meV}$. For $E_{F}=E_{D}$, the QSH effect can persist in a wide range of magnetic field, from zero to about $10 \mathrm{~T}$ (see Fig. 6). If magnetic field $B_{\perp}$ increases further, the spin-up and spin-down gaps overlap, then a gap of the whole band is opened and the system becomes an insulator. At Fermi energy $E_{F}=E_{D}$, the system transits directly from the QSH phase into the insulator phase, where the longitudinal resistance is divergent. At the larger magnetic field, the insulator phase can extend to a wider range of $E_{F}$.

In Figs. 3(c) and 3(d) Fermi energy moves down into the valence bands within $-|M|>E_{F}$. The Hall resistance $R_{H}$ is negative due to the carriers being hole now, and $\left|R_{H}\right|$ linearly increases in small magnetic field $B_{\perp}$. When the magnetic field continues to increase, the quantized Hall-resistance plateaus are shown due to LLs. The plateau values are at $-h / n e^{2}$ with the integer $n=1,2,3, \ldots$ Eventually, the system enters the QAHL phase and the resistance plateaus of $\left(R_{L}, R_{H}\right)=$ $\left(0,-h / e^{2}\right)$ appear, because of the opening of the gap in spin-up subbands. For the lower Fermi energy, the hole density is higher and the system enters the QAHL phase at a larger magnetic field.

In Fig. 6, the phase diagram in the $E_{F}-B_{\perp}$ plane is plotted. There is a four-phase (QSH, insulator, and two QAHL phases) crossing point located at $E_{F}=E_{D}$ and $B_{\perp} \approx 10 \mathrm{~T}$. For $B_{\perp}<$ $10 \mathrm{~T}$, the system can be three phases (one QSH and two QAHL phases) depending on the Fermi energy. It is obvious that the spin-up QAHL phase of $C_{+}=1$ and $C_{-}=0$ emerges as soon as $B_{\perp} \neq 0$. But the spin-down QAHL phase of $C_{+}=0$ and $C_{-}=-1$ has a threshold of magnetic field $B_{c} \approx 0.5 \mathrm{~T}$, and it emerges until the magnetic field $B_{\perp}>B_{c}$. With the increasing of $B_{\perp}$, the width of the QSH region decreases and the widths of two QAHL states increase. For $B_{\perp}>10 \mathrm{~T}$, the QSH phase disappears and the insulator phase occurs because a gap of the whole band is opened. In addition, in the outside of the four

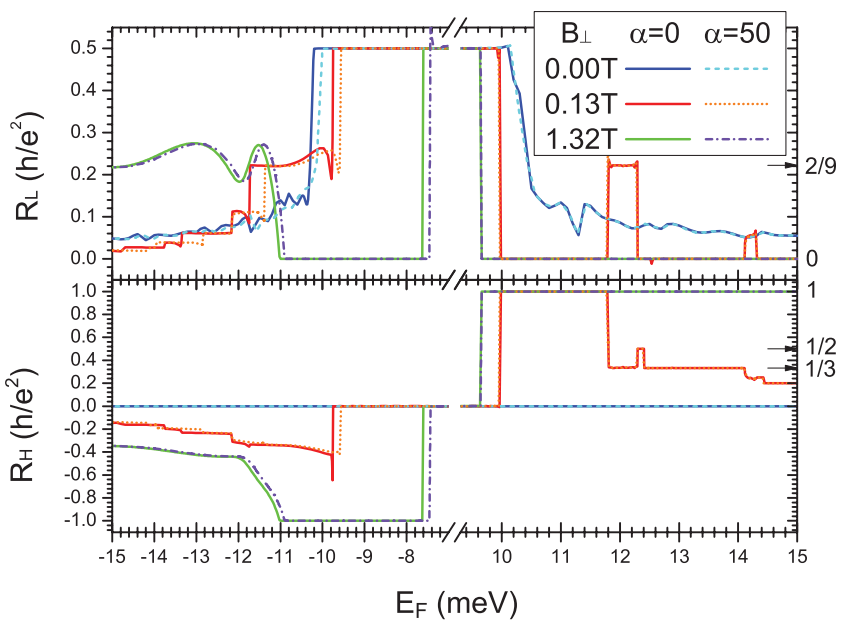

FIG. 7. (Color online) The resistances $R_{L}$ and $R_{H}$ as a function of Fermi energy with Rashba spin-orbit interaction and fixed magnetic field. The Rashba strength is taken by $\alpha=50 \mathrm{meV} \mathrm{nm}$. For comparison, the case of without Rashba spin-orbit interaction, $\alpha=0$, is also shown. In the plots, the curves of $\alpha=50 \mathrm{meV} \mathrm{nm}$ and $\alpha=0$ almost overlap.

phases, the system is in a metal state for the small magnetic field, while it is in the quantum Hall $(\mathrm{QH})$ phase at the large magnetic field.

Let us study the effect of Zeeman splitting on the phase diagram. The Zeeman effect can be considered by $V \rightarrow V+$ $\mu_{B} B_{\perp} \operatorname{diag}\left(g_{E \perp}, g_{H \perp},-g_{E \perp},-g_{H \perp}\right)$ for Eq. (1), where $\mu_{B}$ is the Bohr magneton and the out-of-plane $g$ factors, $g_{E \perp}=22.7$ and $g_{H \perp}=-1.21$, are given for the quantum well thickness $7 \mathrm{~nm} .{ }^{15}$ The similar phase diagram is found in Fig. 6 (see the red dotted curves). All four phases (QSH, insulator, and two QAHL phases) survive well. In particular, the aforementioned four pairs of longitudinal and Hall resistance plateaus at the small magnetic field $\left(B_{\perp} \sim 0.1 \mathrm{~T}\right)$ are almost not affected. On the other hand, due to Zeeman effect, the QSH phase disappears for $B_{\perp}>7.5 \mathrm{~T}$ instead of $B_{\perp}>10 \mathrm{~T}$.

Let us investigate the effect of Rashba spin-orbit interaction on these resistance plateaus (see Fig. 7). We find that the effect of the Rashba term is very weak. On the side of $E_{F}>0$, the plateaus are almost not affected by the Rashba term regardless of the magnetic field. In the QAHL regime, there is only the spin-up edge state and the electron hopping between different spin states cannot occur, so the width of the plateaus $\left(R_{L}, R_{H}\right)=\left(0, h / e^{2}\right)$ is the same as the case of no Rashba interaction [see Fig. 1(c)]. The plateaus of $\left(R_{L}, R_{H}\right)=\left(\frac{2}{9} h / e^{2}, \frac{1}{3} h / e^{2}\right)$ are not changed also, because of the spatial separation of the wave functions of the spin-up edge state $k_{u 1}$ and spin-down edge state $k_{d 1}$ (see Fig. 5). For the plateaus of higher energy, they result from bulk LLs and affected very little by the weak Rashba term. On the side of $E_{F}<0$, at the large magnetic field, the QAHL regime moves lightly to the higher Fermi energy owing to Rashba interaction, but its width is hardly changed, as seen in Fig. 1(c).

At last, the effect of disorder on the resistance plateaus in QSH and QAHL regions is investigated as well in Fig. 8. Here, Anderson on-site disorder is considered and only resides on the region between two cross parts in the inset of Fig. 1(b). Accordingly, in Eq. (1), $V \rightarrow V+\omega_{\mathbf{i}} \operatorname{diag}(1,1,1,1)$ for the 


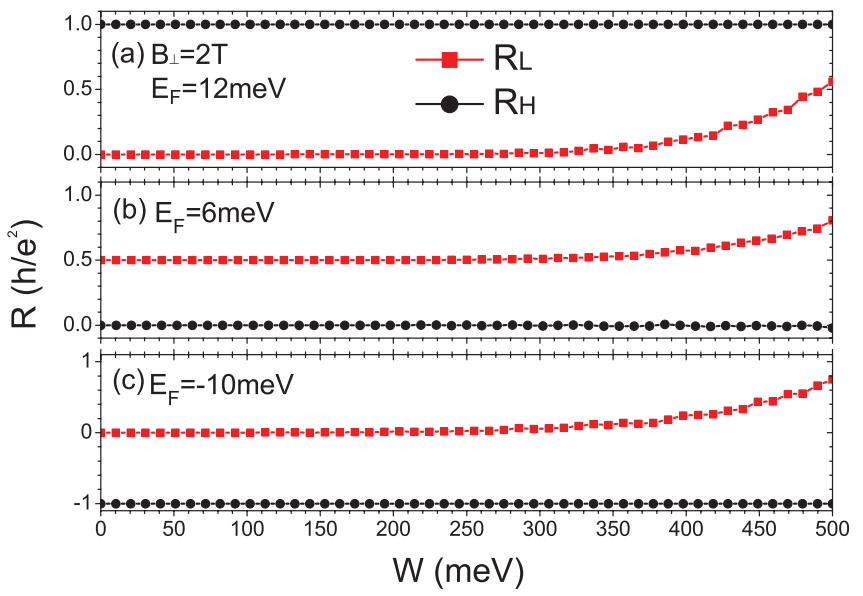

FIG. 8. (Color online) The resistances $R_{L}$ and $R_{H}$ as a function of disorder strength $W$ with fixed magnetic field $B_{\perp}=2 \mathrm{~T}$ and $\alpha=0$. The Fermi energies are selected from three black dots in Fig. 6 with (a) $12 \mathrm{meV}$, (b) $6 \mathrm{meV}$, and (c) $-10 \mathrm{meV}$. Each resistance value is averaged over 500 disorder configurations.

corresponding sites, and the on-site disorder energy $\omega_{\mathbf{i}}$ is uniformly distributed in the range $[-W / 2, W / 2]$ with the disorder strength $W$. For two QAHL states in Figs. 8(a) and 8(c), the Hall resistance plateaus are very robust against the disorder and they can perfectly hold even though $W$ reaches $500 \mathrm{meV}$. The longitudinal resistance plateaus $\left(R_{L}=0\right)$ does not change up to $W=300 \mathrm{meV}$, beyond which they begin to increase. For the QSH state in Fig. 8(b), the longitudinal resistance plateau departs from the plateau $\frac{1}{2} h / e^{2}$ when $W>$ $350 \mathrm{meV}$, and the Hall resistance plateau $R_{H}^{2}=0$ can be kept better than the corresponding longitudinal resistance plateau.
Therefore all these resistance plateaus are well maintained in a large range of disorder strength.

\section{CONCLUSION}

In summary, we have investigated the effect of magnetic field on electron transport in the inverted band structure of the $\mathrm{HgTe} / \mathrm{CdTe}$ quantum well. Although magnetic field breaks time-reversal symmetry, the QSH plateaus of $\left(R_{L}, R_{H}\right)=$ $\left(\frac{1}{2} h / e^{2}, 0\right)$ can still remain until the magnetic field is larger than $10 \mathrm{~T}$. Moreover, due to the gap opening in the spin-up and spindown subbands under the magnetic field, two QAHL phases appear, in which the system has only one spin-up or spin-down edge state. When the magnetic field is very large $(>10 \mathrm{~T})$, the QSH phase disappears finally and an insulator phase emerges. The phase diagram in Fermi energy versus magnetic field plane is given and the longitudinal and Hall resistances are studied. Owing to the QSH and QAHL effects, both longitudinal and Hall resistances exhibit the plateau structures. Besides, in the suitable magnetic field, a pair of exotic resistance plateaus of $\left(R_{L}, R_{H}\right)=\left(\frac{2}{9} h / e^{2}, \frac{1}{3} h / e^{2}\right)$ can occur, in which the edge states with the same spin counterpropagate at the one edge, like the $\frac{2}{3}$ fractional quantum Hall effect. Finally, these plateaus are hardly affected by Rashba spin-orbit interaction, Zeeman effect, and Anderson disorder.

\section{ACKNOWLEDGMENTS}

This work was financially supported by NSF-China under Grants No. 10821403, No. 10974236, and No. 11074174, NBRP of China, and RGC Grant No. HKU 705611P from the government of HKSAR. *sunqf@iphy.ac.cn

${ }^{1}$ C. L. Kane and E. J. Mele, Phys. Rev. Lett. 95, 146802 (2005).

${ }^{2}$ C. L. Kane and E. J. Mele, Phys. Rev. Lett. 95, 226801 (2005).

${ }^{3}$ B. A. Bernevig, T. L. Hughes, and S.-C. Zhang, Science 314, 1757 (2006).

${ }^{4}$ B. A. Bernevig and S.-C. Zhang, Phys. Rev. Lett. 96, 106802 (2006).

${ }^{5}$ H. Jiang, S. G. Cheng, Q.-F. Sun, and X. C. Xie, Phys. Rev. Lett. 103, 036803 (2009).

${ }^{6}$ M. König, S. Wiedmann, C. Brüne, A. Roth, H. Buhmann, L. W. Molenkamp, X.-L. Qi, and S.-C. Zhang, Science 318, 766 (2007).

${ }^{7}$ C. Wu, B. A. Bernevig, and S.-C. Zhang, Phys. Rev. Lett. 96, 106401 (2006).

${ }^{8}$ L. Fu and C. L. Kane, Phys. Rev. B 76, 045302 (2007); H. Zhang, C.-X. Liu, X.-L. Qi, X. Dai, Z. Fang, and S.-C. Zhang, Nat. Phys. 5, 438 (2009).

${ }^{9}$ D. Hsieh, D. Qian, L. Wray, Y. Xia, Y. S. Hor, R. J. Cava, and M. Z. Hasan, Nature (London) 452, 970 (2008); Y. Xia, D. Qian, D. Hsieh, L. Wray, A. Pal, H. Lin, A. Bansil, D. Grauer, Y. S. Hor, R. J. Cava, and M. Z. Hasan, Nat. Phys. 5, 398 (2009); D. Hsieh, Y. Xia, L. Wray, D. Qian, A. Pal, J. H. Dil, J. Osterwalder, F. Meier, G. Bihlmayer, C. L. Kane, Y. S. Hor, R. J. Cava, and M. Z. Hasan, Science 323, 919 (2009); Y. L. Chen, J. G. Analytis, J.-H. Chu,
Z. K. Liu, S.-K. Mo, X. L. Qi, H. J. Zhang, D. H. Lu, X. Dai, Z. Fang, S. C. Zhang, I. R. Fisher, Z. Hussain, and Z.-X. Shen, ibid. 325, 178 (2009).

${ }^{10}$ C.-X. Liu, X.-L. Qi, X. Dai, Z. Fang, and S.-C. Zhang, Phys. Rev. Lett. 101, 146802 (2008).

${ }^{11}$ R. Yu, W. Zhang, H.-J. Zhang, S.-C. Zhang, X. Dai, and Z. Fang, Science 329, 61 (2010).

${ }^{12}$ Y. Yang, Z. Xu, L. Sheng, B. Wang, D. Y. Xing, and D. N. Sheng, Phys. Rev. Lett. 107, 066602 (2011).

${ }^{13}$ X. C. Zhang, A. Pfeuffer-Jeschke, K. Ortner, V. Hock, H. Buhmann, C. R. Becker, and G. Landwehr, Phys. Rev. B 63, 245305 (2001).

${ }^{14}$ Y. S. Gui, C. R. Becker, N. Dai, J. Liu, Z. J. Qiu, E. G. Novik, M. Schäfer, X. Z. Shu, J. H. Chu, H. Buhmann, and L. W. Molenkamp, Phys. Rev. B 70, 115328 (2004).

${ }^{15}$ M. König, H. Buhmann, L. W. Molenkamp, T. Hughes, C.-X. Liu, X.-L. Qi, and S.-C. Zhang, J. Phys. Soc. Jpn. 77, 031007 (2008).

${ }^{16}$ D. G. Rothe, R. W. Reinthaler, C. X. Liu, L. W. Molenkamp, S. C. Zhang, and E. M. Hankiewicz, New J. Phys. 12, 065012 (2010).

${ }^{17}$ O. Mauritz and U. Ekenberg, Phys. E 6, 779 (2000).

${ }^{18}$ H. Jiang, L. Wang, Q.-F. Sun, and X. C. Xie, Phys. Rev. B 80, 165316 (2009).

${ }^{19}$ Q.-F. Sun, Y.-X. Li, W. Long, and J. Wang, Phys. Rev. B 83, 115315 (2011). 
${ }^{20}$ J. Maciejko, X.-L. Qi, and S.-C. Zhang, Phys. Rev. B 82, 155310 (2010).

${ }^{21}$ S. Datta, Electronic Transport in Mesoscopic Systems (Cambridge University Press, Cambridge, England, 1995).

${ }^{22}$ M. P. L. Sancho, J. M. L. Sancho, and J. Rubio, J. Phys. F 15, 851 (1985).
${ }^{23}$ G. Tkachov and E. M. Hankiewicz, Phys. Rev. Lett. 104, 166803 (2010).

${ }^{24}$ M. D. Johnson and A. H. MacDonald, Phys. Rev. Lett. 67, 2060 (1991).

${ }^{25}$ C. L. Kane and M. P. A. Fisher, Phys. Rev. B 55, 15832 (1997). 